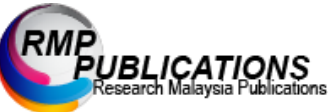

Journal of Engineering and Science Research 5 (2): 01-05, 2021

e-ISSN: 2289-7127

(C) RMP Publications, 2021

DOI: $10.26666 / \mathrm{rmp} . j e s r .2021 .2 .1$

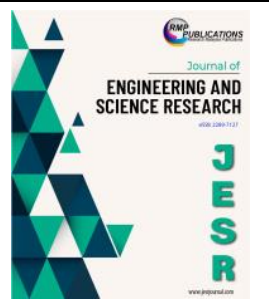

\title{
The Concept of Miniaturized Fiber-Optic Surface Plasmon Resonance Biosensor for In Situ Viral Detection
}

\author{
Sharifah Norsyahindah Syed Nor ${ }^{l}$ Nur Syafiqah Rasanang ${ }^{l}$ Salmah Karman ${ }^{1,3}$ Wan Safwani Wan Kamarul \\ Zaman $^{l}$ Sulaiman Wadi Harun ${ }^{2}$ and Hamzah Arof ${ }^{2}$ \\ ${ }^{1}$ Department of Biomedical Engineering, Faculty of Engineering, \\ University of Malaya, 50603, Kuala Lumpur, Malaysia \\ ${ }^{2}$ Department of Electrical Engineering, Faculty of Engineering, \\ University of Malaya, 50603, Kuala Lumpur, Malaysia \\ ${ }^{3}$ Centre of Advanced Manufacturing and Material Processing, Faculty of Engineering, \\ University of Malaya, 50603, Kuala Lumpur, Malaysia
}

\begin{abstract}
The surface plasmon resonance (SPR) technique has been incorporated in advancing numerous fields such as biology, biochemistry, molecular interactions monitoring, and medical diagnostics. For the past thirty years, researchers utilized SPR biosensing for rapid detection of viral infection. This paper demonstrates the concept of miniaturization of SPR for in-situ early screening of viral infection. The fundamental principles, including from the total internal reflection, evanescent wave, surface plasmons and surface plasmon oscillations are summarized and highlighted. The integration of miniaturized fiber-optic SPR biosensor with mobile phone device suitable for future in-situ screening. The sensor was fashioned by a light-guiding silica capillary coated with gold $(\mathrm{Au})$ thin film as a sensing layer. The flash of the phone act as the light source, and the camera act as the detector where it captures change of light intensity. The captured images are then analyzed using a simple software application installed on the phone. The potential of an advanced miniaturized system as an alert system for viral recognition in public areas was also overviewed in this paper. By implementing the real-time alert system of viral infection in public places, the management of disease transmission could be improved.
\end{abstract}

Keywords: Surface Plasmon Resonance, Fiber-Optic Plasmonic Biosensor, Point-of-care device, Smartphone $\underline{\text { SPR-Based Biosensor, Virus Detection }}$

\section{INTRODUCTION}

Over the past few decades, surface plasmon resonance biosensing has captured the attention of investigators, especially in the field of biology [1], biochemistry, medical diagnostics [2], biomarker detection [3], and medical sciences due to its ability of label-free, realtime and non-invasive technology. SPR technology can be utilize in biomolecular interactions monitoring such as DNA hybridization and protein bindings process [4, 5]. The phenomenon was first observed in 1902 [6], and not until 1983 an SPR-based sensor was developed to monitor biomolecular interactions [7]. Numerous literature demonstrate SPR as a suitable alternative technique for diagnostics devices, pathogen recognition, and others $[8,9]$.

The phenomenon involves the changes of refractive index (RI) on the metallic sensing layer for example gold, silver or aluminum, at the interfaces of two various media such as glass and liquid. The RI changes initiated when the immobilized ligand captures the target analyte injected into the system. As the target analyte interacts with the immobilized ligand, the RI nearest to the surface deviates. The light detector will receive and evaluate the shift of intensity, generating an SPR signal. The conventional SPR setup is called the Kretschmann configuration, where it uses a thin layer of metal film coated on top of a prism surface [10]. However, prism-based SPR sensor is bulky and

Corresponding Author: Salmah Karman, Department of Biomedical Engineering, Faculty of Engineering, University of Malaya, 50603, Kuala Lumpur, Malaysia, +603-7967 7694, salmah_karman@um.edu.my 
unable to be used for remote sensing applications. Whereas, fiber-optic SPR sensor can be miniatured where remote sensing is made possible. Light passing through a medium will be attenuated and be evaluated by complex refractive index, $\mathrm{n}_{\mathrm{k}}^{2}$ also known as complex dielectric function. It consists of two parts, real and imaginary. $\mathrm{n}_{\mathrm{k}}^{2}$ is used to determine the reliability of a thin film. For the metal film sensing layer, either gold $(\mathrm{Au})$ or silver $(\mathrm{Ag})$ is usually considered [1].

Presently, despite practicing a strict standard operating procedure (SOP) established by the government, infected cases among the community still arise with a new cluster developed. Public places such as the school, workplace, gas station, and supermarket are the places where most of the community goes to and expose to the virus through direct or indirect contact. To solve this, high technology screening devices for airborne viruses capable of providing real-time response are required to monitor public places. Herein, we proposed the miniaturized fiber-optic SPR device that can be incorporated in mobile phones where people bring it everywhere for on-site monitoring. Additionally, the proposed device can be installed in various public areas on the wall, seat, door, and numerous others to alert the community of their safety and health status.

\section{FUNDAMENTAL PRINCIPLE OF SPR}

Light beam originated from the light source illuminating the metallic sensing layer at a certain angle. It refracted closer to the surface as it moved from a denser to less dense medium. As mentioned by Pluchery et al., the utilized light source should be monochromatic and ppolarized, where the light is polarized in the plane of the surface to acquire an excellent sharp dip [11]. The light beam conjured at a certain incidence angle containing photon interacts with the electron at metal surface generating resonating electrons, also known as surface plasmons (SP). As the incident angle is adjusted to make all the light illuminate the interface, total internal reflection (TIR) occurs. Herein, TIR angle is called the resonance angle, where the incident angle $(\Theta)$ is bigger than the critical angle.

When TIR occurs, the reflected photons produce an electric lot on the opposite side of the interface. Then the plasmons will generate a comparable field that reaches into the medium on either side of the metallic sensing layer called the evanescent wave. This type of wave can improve using a gold film with optimum thickness placed on the interface. As a result, the wave goes through the gold thin film from a denser medium to less dense medium. The oscillations of the electrons generate a wave pattern spatial distribution of the particles called the surface plasmon wave (SPW), also known as surface plasmon polaritons (SPPs). The light energy-containing plasmons is reflected, and absorbs the power of the plasmons. It transforms into reflected light received by the light detector.

An alternative to bulky prism-based SPR sensors, fiberoptic have been proven to be helpful in miniature SPR sensors. Fiber-optic SPR sensors has excellent advantages for in situ measurements due to its low fabrication cost, simplest structure, and smaller sample volume $[12,13]$. The ultimate advantage fiber optic is the SPR probe can be miniaturized. Zhao et al. [14] demonstrate a fiber-optic SPR sensor for rapid influenza detection. The middle part of the fiber optic exposed followed by deposition of the gold film. Then, the sensing layer is functionalized with the immobilization of antibodies. If the airborne virus is captured by the immobilized antibodies, a signal proportional to the biomolecule concentration is produced, alerting the users.

\section{METHODOLOGY: MINIATURIZED FIBER- OPTIC SPR BIOSENSOR}

Several miniaturized SPR biosensors [15] were developed for environmental monitoring and bacterial recognition [16, 17]. For example, [18] develop an SPR smartphone biosensor using a disposable sensor ship, gold diffraction grating, and compact disk (CD) that depends on the built-in flashlight source and camera. The developed device provides label-free, real-time analysis and enable high-throughput screening in an array format. Also, it can be connected or dismantled from smartphones easily. To eliminate the usage of the bulky prism, a fiber-optic structure is introduced in the proposed SPR system. The sensing layer consists of an optical fiber, and thin metal.

In the proposed setup, each layer is characterized by its dielectric constant, $\varepsilon_{\mathrm{k}}$, and magnetic permeability, $\mu_{\mathrm{k}}$. The first layer is the core of the fiber, followed by the thin metal. For each layer, we determine the complex optical index $\mathrm{n}_{\mathrm{k}}^{2}$ connected to the dielectric constant by:

$$
n_{k}^{2}=\varepsilon_{k}=\left(n_{k}+i k_{k}\right)^{2}
$$

Where the real part, $n_{k}$ is the index of refraction and the imaginary part, $\mathrm{k}_{\mathrm{k}}$ is the extinction coefficient. The complex optical index for $\mathrm{Au}, \mathrm{Ag}$, and $\mathrm{Cu}$ are calculated, and compared to determine the optimum setup for the proposed sensor. The real part of the dielectric constant represents the shifting of the resonance curve. In contrast, the imaginary part of the dielectric constant is the sharpness of the resonance curve. 
Fiber-optic SPR sensors possess significant superiority, for in situ sensing. In addition, it eradicates the usage of bulky, expensive optical components, for example, the prisms, slides including the mechanical parts. Instead, the core of the optical fiber act as a prism from a typical SPR configurations. The sensing elements and optical components of the sensor were linked by optical fibers. They were fixed on a lightweight phone case where they can be installed and removed easily. The cladding is removed, and the silica capillaries are coated with $53 \mathrm{~nm}$ gold film followed by a dielectric sensing layer to be utilized as a sensing element of the sensor. The sensing layer is packaged into the flow cell, making the assembly and dismantling process of the device troublefree. The flash and phone camera that acts as a light source strikes one end of the fiber, producing and evanescent field where it excites the surface plasmons at the metal-dielectric interface. A narrow-band filter (center wavelength of $590 \mathrm{~nm}$ and full width at halfmaximum (FWHM) of $8 \mathrm{~nm}$ ) placed between the flash of the cell phone and the lead-in fibers provides nearly monochromatic incident light. Once the sample is injected into the flow cell, SPR resonance causes the light interacting with the sensing region absorbed by the camera. The camera then measures the intensity of light change.

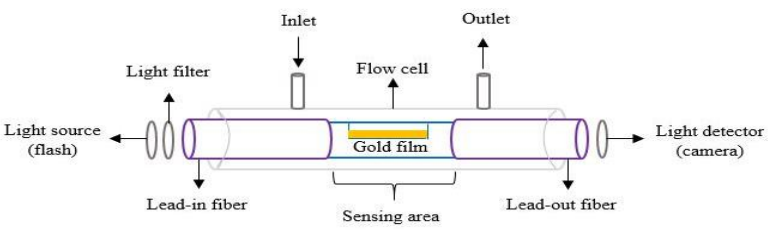

Figure 1 Schematic diagram of the proposed fiber-optic SPR sensor. Modified from Liu et al. [19].

Figure 1 illustrates the setup of the proposed sensor. A step-index multi-mode fiber constitutes fused silica core is considered suitable for applications that need high power densities, such as medical power delivery. The core-cladding structure of the optical fiber aids the electromagnetic wave inside the fiber to undergo total internal reflection, similar to the fundamental of prismbased SPR sensor. The principle of the SPR sensor incorporated into smartphone depends on the penetration of the filter by the light from the smartphone flash, and transmission to the camera through inlet fibers. Since the sensor are installed on the backside of a phone case, the front side of the phone consist of the touch screen interface and the display are not affected throughout the virus detection operation.

Functionalization of sensing layer is performed with amine-coupling reaction to aids immobilization of ligand. Once the ligands are immobilized, the sensing layer is assembled in the flow cell in line with the leadin and lead-out fibers. The lead-in fibers are aligned and connected to the light source, whereas the lead-out fibers are aligned and connected to the light detector. Both the lead-in and lead-out fibers are wrapped in a black rubber tube to avoid light drifting away. The light entering the lead-in fibers from the phone's flash illuminates the SPR system, whereas the light from end faces of the lead-out fibers is detected by the phone's camera. The sensing layer are sealed to avoid contamination and exposure and only the inlet fiber is open to virus pumped into the flow cell. To put it in simpler terms, the flash act as light source and the camera act as a detector.

If the airborne virus is present, the immobilized antibody on the sensing layer will capture the virus. The interaction between virus and immobilized antibody induces intensity change of the light to emerge from the outlet fiber measured by the phone camera. The images of a light intensity changes captured by the camera at $2 \mathrm{~Hz}$ frequency were transmitted to a software application. The camera exposure, flash activation, and light intensity images are controlled from the application. The change in light intensity due to the binding interactions between the immobilized ligand and the virus sample was recorded and plotted as a function of time through the application. The application is capable of signaling the user of high-risk public places where airborne viruses exist. The proposed sensor can be utilized in a few different platforms, for instance, iPhones, and Android phones.

\section{RESULT AND DISCUSSION}

Based on the Lorentz-Drude model, the complex optical index of $\mathrm{Au}, \mathrm{Ag}$ and $\mathrm{Cu}$ at $590 \mathrm{~nm}$ incident light is demonstrated in table 1 . After computing the value of $\mathrm{n}_{\mathrm{k}}{ }^{2}$ we will evaluate the real, and imaginary part of the dielectric constant.

Table 1: Index of refraction, extinction coefficient and complex optical index for $\mathrm{Au}, \mathrm{Ag}$, and $\mathrm{Cu}$ at $590 \mathrm{~nm}$ incident light.

\begin{tabular}{|l|c|c|c|}
\hline Type of metal & $\mathrm{Au}$ & $\mathrm{Ag}$ & $\mathrm{Cu}$ \\
\hline $\begin{array}{l}\text { Index of } \\
\text { refraction, } \mathbf{n}_{\mathbf{k}}\end{array}$ & 0.38216 & 0.13817 & 0.42094 \\
\hline $\begin{array}{l}\text { Extinction } \\
\text { coefficient, } \mathbf{k}_{\mathbf{k}}\end{array}$ & 2.7561 & 3.4838 & 3.1437 \\
\hline $\begin{array}{l}\text { Complex } \\
\text { optical index, } \\
\mathbf{n}_{\mathbf{k}}{ }^{2}\end{array}$ & \begin{tabular}{l}
$-7.4500+$ \\
\hline
\end{tabular} & $\begin{array}{l}-12.1178 \\
+0.9627\end{array}$ & $\begin{array}{l}-9.7067+ \\
2.6466 \mathrm{i}\end{array}$ \\
\hline
\end{tabular}

Based on reported literature, the sharpness of the resonance curve depends on the imaginary part of the dielectric constant [1]. From fig. 2, we can see that $\mathrm{Cu}$ exhibit the highest value of the imaginary part compare to $\mathrm{Au}$ and $\mathrm{Ag}$. Hence, $\mathrm{Cu}$ has a narrower SPR curve with 
excellent detection accuracy. But $\mathrm{Cu}$ is prone to oxidation if it stands alone, and $\mathrm{Ag}$ is not stable in chemical medium. Consequently, Au is more suitable to be used as the metal film in the sensing area due to its biocompatibility and stability in a chemical medium $[20$, 21].

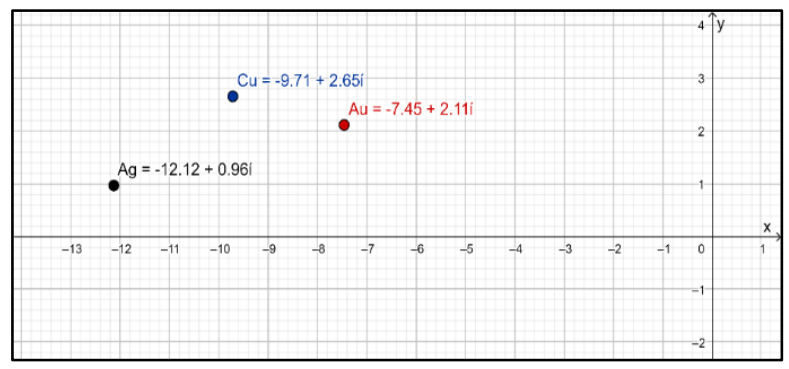

Figure 2 Graph of imaginary versus real part of dielectric constant

The real part of the dielectric constant illustrates the shift of the resonance curve. Au displays the highest value of the real part among $\mathrm{Ag}$ and $\mathrm{Cu}$. Hence, it supports the fact that $\mathrm{Au}$ provide more shifting in the resonance parameter to change in refractive index of sensing layer [1]. According to the literature review, the sensing layer made from $\mathrm{Au}$ offer more stability and compatibility than $\mathrm{Ag}$. and $\mathrm{Cu}$ [21]. Hence, $\mathrm{Au}$ was chosen as the sensing layer with a suitable value of the imaginary dielectric function.

\section{CONCLUSION AND OUTLOOK}

The development of SPR sensors has driven biomedical, medical diagnostics, food, toxic detection, and other fields. SPR optical sensor operates based on photonic excitation. Hence, choosing the most suitable light source is crucial because, without the excellent characteristics of the light source, other superb aspects of the sensor will not be functioning correctly. In addition, the metal film must exhibit vast conduction band electrons proficient in resonating with the incoming photon from the light source at a fitted wavelength. For example, gold demonstrates excellent resistance to oxidation, increasing the lifespan of the sensor.

For the past thirty years, SPR biosensing has experienced a breakthrough in real-time monitoring application of molecular interactions [22]. Lately, this application has been extensively studied for the advancement of a prompt diagnostic technique for viral infection [23, 24]. SPR-based biosensors demonstrate excellent feature towards a rapid, highly specific, and sensitive for early recognition of viral infections. The characteristic of SPR sensor that enables label-free, realtime monitoring [25] and quick [26] making it suitable to be utilized as part of diagnostics technique for viral infections before it progresses to a pandemic. Fiberoptic SPR sensor can be miniatured, which is highly useful for swift viral recognition. The sensor excludes the use of bulky prism to create an evanescent field to realize the SPR phenomenon.

Data from table 1 shows that $\mathrm{Cu}$ exhibits excellent detection accuracy. However, $\mathrm{Cu}$ is unstable and prone to corrosion. In contrast, the value of the real part of the dielectric constant for $\mathrm{Au}$ is the highest, which represent better shifting in resonance curve. Au is suitable to be used as a sensing layer from the value of the complex optical index and its superior characteristics that include stability and biocompatibility [20, 21]. Henceforward, Au can be coupled with other metals such as highly sensitive Ag in a bi-metallic configuration to enhance its sensitivity.

In the future, the developed device can be in the form of a miniature device without the utilization of smartphones to be installed in various public zones where it will signal the community of high-risk places. To manufacture the device in large quantities, the cost of the SPR components, miniaturization process, design, and beautification should be considered. An alternative power source where it exhibits low consumption is an attractive substitute. Once the miniature SPR sensor manages to be fabricated, the device can be installed in public areas such as the airport as an early screening system to contain pandemic outbreaks Also, the sensor can be used as an alert system in schools, supermarkets, and gas stations where the community goes to daily. The proposed sensor is portable and capable of providing swift, early detection where pandemic management is crucial. It can be use in various applications such as health, medicine and environmental monitoring.

\section{ACKNOWLEDGMENTS}

The authors acknowledge the financial support from Fundamental Research Grant Scheme (FRGS), [FP1142020] and University Malaya Research Grant (UMRG), [IIRG002B-19SAH].

\section{REFERENCES}

[1] Bolduc, O., P. Lambert-Lanteigne, D. Colin, S. Zhao, C. Proulx, D. Boeglin, W. Lubell, J. Pelletier, J. Fethiere, H. Ong and J.-F. Masson (2011). Modified peptide monolayer binding Histagged biomolecules for small ligand screening with SPR biosensors. The Analyst 136, 31423148.

[2] Damborský, P., J. Švitel and J. Katrlík (2016). Optical biosensors. Essays in biochemistry 60(1), 91-100.

[3] Grasso, G., R. D'Agata, E. Rizzarelli, G. Spoto, L. D'Andrea, C. Pedone, A. Picardi, A. Romanelli, M. Fragai and K. J. Yeo (2005). Activity of anchored human matrix 
metalloproteinase-1 catalytic domain on $\mathrm{Au}$ (111) surfaces monitored by ESI-MS. J Mass Spectrom 40(12), 1565-1571.

[4] Gupta, B. and R. K. Verma (2009). Surface Plasmon Resonance-Based Fiber Optic Sensors: Principle, Probe Designs, and Some Applications. Journal of Sensors 2009.

[5] Homola, J. (2003). Present and future of surface plasmon resonance biosensors. Anal Bioanal Chem 377(3), 528-539.

[6] Homola, J. (2008). Surface plasmon resonance sensors for detection of chemical and biological species. Chem Rev 108(2), 462-493.

[7] Hossain, M. B. and M. M. Rana (2016). Graphene Coated High Sensitive Surface Plasmon Resonance Biosensor for Sensing DNA Hybridization. Sensor Letters 14, 145-152.

[8] Kim, S. A., S. J. Kim, H. Moon and S. B. Jun (2012). In vivo optical neural recording using fiber-based surface plasmon resonance. Optics Letters 37(4), 614-616.

[9] Klotz, M., S. Opper, K. Heeg and S. Zimmermann (2003). Detection of Staphylococcus aureus enterotoxins A to D by real-time fluorescence PCR assay. Journal of clinical microbiology 41(10), 4683-4687.

[10] Kretschmann, E. and H. Raether (1968). Notizen: Radiative Decay of Non Radiative Surface Plasmons Excited by Light. Zeitschrift für Naturforschung A 23.

[11] Liu, Y., Q. Liu, S. Chen, F. Cheng, H. Wang and W. Peng (2015). Surface Plasmon Resonance Biosensor Based on Smart Phone Platforms. Scientific reports 5, 12864.

[12] Marchesini, G. R., K. Koopal, E. Meulenberg, W. Haasnoot and H. Irth (2007). Spreeta-based biosensor assays for endocrine disruptors. Biosens Bioelectron 22(9-10), 1908-1915.

[13] Martins e Silva, J. (1991). Biochemical characterization and metabolic effects of tumor necrosis factor. Acta Med Port 4 Suppl 1, 20s-27s.

[14] Nguyen, H. H., J. Park, S. Kang and M. Kim (2015). Surface plasmon resonance: a versatile technique for biosensor applications. Sensors (Basel) 15(5), 10481-10510.

[15] Pluchery, O., R. Vayron and K.-M. Van (2011). Laboratory experiments for exploring the surface plasmon resonance. European Journal of Physics 32, 585.

[16] Prabowo, B. A., R. Y.-L. Wang, K. Secario, P.-T. Ou, A. Alom, J.-J. Liu and K.-C. Liu (2017). Rapid Detection and Quantification of Enterovirus 71 by a Portable Surface Plasmon Resonance Biosensor. Biosensors \& Bioelectronics 92, 186-191.

[17] Qu, J.-H., A. Dillen, W. Saeys, J. Lammertyn and D. Spasic (2020). Advancements in SPR biosensing technology: An overview of recent trends in smart layers design, multiplexing concepts, continuous monitoring and in vivo sensing. Analytica Chimica Acta 1104, 10-27.

[18] Saad, Y., M. Selmi, M. H. Gazzah, A. Bajahzar and H. Belmabrouk (2019). Performance enhancement of a copper-based optical fiber SPR sensor by the addition of an oxide layer. Optik 190, 1-9.

[19] Widoretno, A. Sjahrurachman, B. E. Dewi, K. Lischer, D. K. Pratami, D. Flamandita and M. Sahlan (2020). Surface plasmon resonance analysis for detecting non-structural protein 1 of dengue virus in Indonesia. Saudi journal of biological sciences 27(8), 1931-1937.

[20] Yanase, Y., A. Araki, H. Suzuki, T. Tsutsui, T. Kimura, K. Okamoto, T. Nakatani, T. Hiragun and M. Hide (2010). Development of an optical fiber SPR sensor for living cell activation. Biosensors and Bioelectronics 25(5), 1244-1247.

[21] Yanase, Y., T. Hiragun, K. Ishii, T. Kawaguchi, T. Yanase, M. Kawai, K. Sakamoto and M. Hide (2014). Surface plasmon resonance for cell-based clinical diagnosis. Sensors (Basel, Switzerland) 14(3), 4948-4959.

[22] Yi, R.-m., Z. Zhang, C.-x. Liu and Z.-m. Qi (2020). Gold-silver alloy film based surface plasmon resonance sensor for biomarker detection. Materials Science and Engineering: C $116,111126$.

[23] Zeng, S., D. Baillargeat, H.-P. Ho and K.-T. Yong (2014). Nanomaterials enhanced surface plasmon resonance for biological and chemical sensing applications. Chemical Society Reviews 43, 3426-3452.

[24] Zhang, J., I. Khan, Q. Zhang, X. Liu, J. Dostalek, B. Liedberg and Y. Wang (2018). Lipopolysaccharides detection on a gratingcoupled surface plasmon resonance smartphone biosensor. Biosens Bioelectron 99, 312-317.

[25] Zhao, S. S., N. Bukar, J. L. Toulouse, D. Pelechacz, R. Robitaille, J. N. Pelletier and J. F. Masson (2015). Miniature multi-channel SPR instrument for methotrexate monitoring in clinical samples. Biosens Bioelectron 64, 664670.

[26] Zhao, X., Y. C. Tsao, F. J. Lee, W. H. Tsai, C. H. Wang, T. L. Chuang, M. S. Wu and C. W. Lin (2016). Optical fiber sensor based on surface plasmon resonance for rapid detection of avian influenza virus subtype H6 Initial studies. J Virol Methods 233, 15-22. 